TO APPEAR IN THE ASTROPHYSICAL JOURNAL LETTERS

Preprint typeset using $\mathrm{LT}_{\mathrm{E}} \mathrm{X}$ style emulateapj v. 08/13/06

\title{
AN OFFSET SEYFERT 2 NUCLEUS IN THE MINOR MERGER SYSTEM NGC 3341
}

\author{
Aaron J. BARth ${ }^{1}$, Misty C. Bentz ${ }^{1}$, Jenny E. Greene ${ }^{2,3}$, And Luis C. Ho ${ }^{4}$ \\ to appear in The Astrophysical Journal Letters
}

\begin{abstract}
We present the discovery of a triplet of emission-line nuclei in the disturbed disk galaxy NGC 3341, based on archival data from the Sloan Digital Sky Survey and new observations from the Keck Observatory. This galaxy contains two offset nuclei within or projected against its disk, at projected distances of 5.1 and $8.4 \mathrm{kpc}$ from its primary nucleus and at radial velocity separation of less than $200 \mathrm{~km} \mathrm{~s}^{-1}$ from the primary. These appear to be either dwarf ellipticals or the bulges of low-mass spirals whose disks have already been stripped off while merging into the primary galaxy. The inner offset nucleus has a Seyfert 2 spectrum and a stellar velocity dispersion of $70 \pm 7 \mathrm{~km} \mathrm{~s}^{-1}$. The outer offset nucleus has very weak emission lines consistent with a LINER classification, and the primary nucleus has an emission-line spectrum close to the boundary between LINER/H II composite systems and H II nuclei; both may contain accreting massive black holes, but the optical classifications alone are ambiguous. The detection of an offset active nucleus in NGC 3341 provides a strong suggestion that black hole accretion episodes during minor mergers can be triggered in the nuclei of dwarf secondary galaxies as well as in the primary.

Subject headings: galaxies: active — galaxies: individual (NGC 3341) — galaxies: interactions — galaxies: nuclei - galaxies: Seyfert
\end{abstract}

\section{INTRODUCTION}

In the standard hierarchical merger paradigm for galaxy formation and evolution, a natural consequence of the merger of two galaxies containing massive black holes is that the two black holes will sink to the center of the host galaxy by dynamical friction, eventually forming a binary and merging together (see Merritt \& Milosavljević 2005 for a review). As a result, there is a general expectation that some merging and post-merger galaxies should have two massive black holes. However, definitive observational identification of galaxies containing two (or more) massive black holes is limited to objects showing evidence for accretionpowered activity from spatially resolved active galactic nuclei (AGNs) (Komossa 2003a). A galaxy with two widely separated active nuclei is often referred to as a "dual AGN" to distinguish it from the case of a "binary AGN" in which the two black holes form a tight, gravitationally bound binary. Several examples of dual AGNs are now known (e.g., Owen et al. 1985; Komossa et al. 2003b; Ballo et al. 2004; Guainazzi et al. 2005; Gerke et al. 2007; Bianchi et al. 2008), most of which are found in major mergers of massive galaxies. It is also expected that minor mergers can trigger episodes of nuclear activity (Hernquist \& Mihos 1995), and dual AGNs could also be present in some minor mergers with dwarf secondary galaxies if the secondary galaxies also harbor central black holes. However, the fraction of dwarf or low-mass galaxies that contain black holes is unknown, and only weakly constrained by AGN surveys (Greene \& Ho 2007) and by stellar-dynamical searches for black holes in dwarf galaxies (Valluri et al. 2005).

In this Letter, we present the discovery of a triplet of emission-line nuclei (at several kpc separation) in NGC 3341.

\footnotetext{
${ }^{1}$ Department of Physics and Astronomy, 4129 Frederick Reines Hall, University of California, Irvine, CA 92697-4575; barth@uci.edu

2 Department of Astrophysical Sciences, Princeton University, Princeton, NJ 08544

${ }^{3}$ Hubble Fellow and Princeton-Carnegie Fellow

4 The Observatories of the Carnegie Institution of Washington, 813 Santa Barbara Street, Pasadena, CA 91101
}

This system appears to contain two dwarf galaxies merging with a massive disk galaxy, and one of the offset nuclei has a Seyfert 2 spectrum. NGC 3341 is therefore a rare example of a disk galaxy with an offset AGN, and an excellent nearby case study for investigations of AGN triggering during a minor merger. The primary nucleus and the second offset nucleus have optical spectra that indicate possible low-level AGN activity as well, making this a candidate dual or even triple AGN system.

\section{ARCHIVAL SDSS DATA}

In the course of searching the Sloan Digital Sky Survey (SDSS) archives for dwarf galaxies with Seyfert-type spectra, we came across the object SDSS J104232.05+050241.9. Its SDSS spectrum is that of a Seyfert 2 nucleus, with strong narrow emission lines superposed on a starlight-dominated continuum. Its $g$-band apparent magnitude is $18.30 \mathrm{mag}$; at $z=0.0271$ (or $D_{L} \approx 117 \mathrm{Mpc}$ for $H_{0}=71 \mathrm{~km} \mathrm{~s}^{-1} \mathrm{Mpc}^{-1}$, $\Omega_{M}=0.27$, and $\Omega_{\Lambda}=0.73$ ), this corresponds to only $M_{g}=-17$ mag. In the online MPA/JHU catalog ${ }^{5}$ of AGNs in SDSS Data Release 4 (Kauffmann et al. 2003), it is listed as an AGN with a host galaxy stellar mass of $1.6 \times 10^{9} M_{\odot}$ and an [O III] $\lambda 5007$ luminosity of $2 \times 10^{6} L_{\odot}$. This object initially appeared to be an excellent example of an AGN in a dwarf host galaxy, but when we examined the SDSS image of the object, we found that it is not an individual dwarf galaxy. Instead, it is an off-nuclear knot within the disk of the larger galaxy NGC 3341. The SDSS catalog magnitude of $g=18.30 \mathrm{mag}$ corresponds only to the brightness of this off-nuclear source, while NGC 3341 overall is a giant disk galaxy with $B=14.9$ $\operatorname{mag}$ (or $M_{B}=-20.3 \mathrm{mag}$ ).

Figure 1 shows the SDSS image of NGC 3341. It is a disturbed disk galaxy (Hubble type $\sim \mathrm{Sa}-\mathrm{Sb}$, but listed in the UGC catalog as "peculiar") undergoing a minor merger, with two dwarf companions within (or projected against) the galaxy disk. We label these off-nuclear sources as NGC 3341B and NGC 3341C, with object B corresponding to

\footnotetext{
${ }^{5}$ http://www.mpa-garching.mpg.de/SDSS
} 


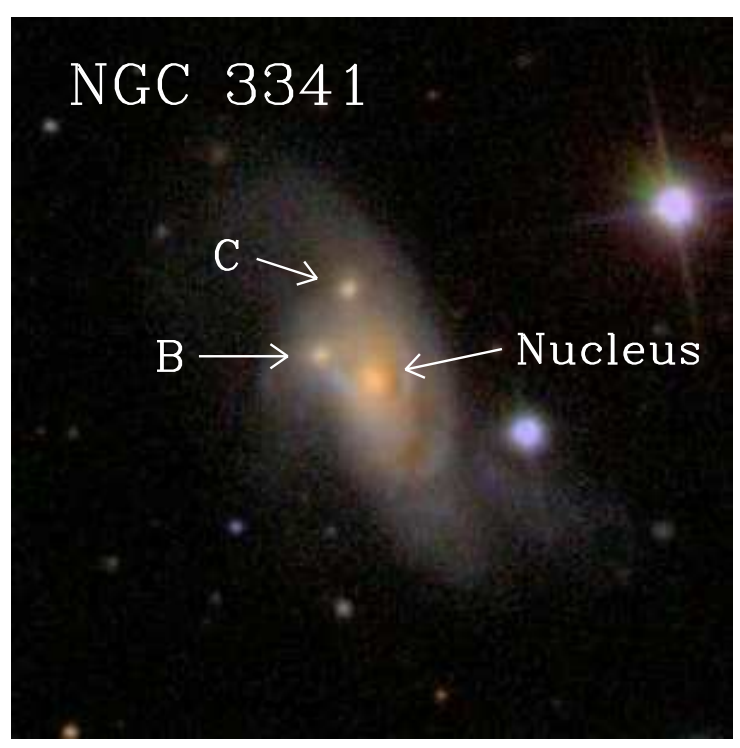

FIG. 1.- SDSS color-composite image of NGC 3341. The field of view is $2^{\prime} \times 2^{\prime}$. North is up and east is to the left.

SDSS J104232.05+050241.9. The SDSS only obtained a spectrum of object B, and not of the galaxy's primary nucleus or of object $\mathrm{C}$. The luminosities of the two off-nuclear sources $\left(M_{g}=-17\right.$ and -16.6 mag for objects B and C) are too high for these to be super star clusters, but they could be dwarf ellipticals, or the bulges of low-mass spirals whose disks have already been stripped off during the merger. Objects B and $\mathrm{C}$ are located at projected separations of 9.'5 and $15 .^{\prime \prime} 6$ (5.1 and $8.4 \mathrm{kpc}$ ) from the primary nucleus, respectively, and the separation between $\mathrm{B}$ and $\mathrm{C}$ is $11 . ! 9(6.4 \mathrm{kpc})$.

\section{KECK OBSERVATIONS}

We obtained spectra of all three nuclei at the Keck-II telescope using the ESI spectrograph (Sheinis et al. 2002) on the night of 2008 March 2 UT. Observing conditions were relatively poor for Mauna Kea, with some cirrus overhead and seeing of $\sim 1 . " 5$. Despite the poor seeing, a narrow slit of width $0 . / 75$ was used in order to resolve narrow absorption and emission lines in dwarf galaxies. The spectra cover 3800 $10900 \AA$ over 10 echelle orders, at a uniform scale of $11.5 \mathrm{~km}$ $\mathrm{s}^{-1}$ pixel $^{-1}$ and instrumental dispersion of $\sigma_{i}=22 \mathrm{~km} \mathrm{~s}^{-1}$. The spectra were obtained with the slit oriented at $\mathrm{PA}=115^{\circ}$, close to the parallactic angle for the midpoint of the exposure sequence, at airmass 1.2-1.3. Exposure times were $900 \mathrm{~s}$ each for the primary nucleus and object C, and $1200 \mathrm{~s}$ for object $\mathrm{B}$. Spectra were extracted using a width of $2^{\prime \prime}$ and flux-calibrated using observations of the standard star Feige 34. The $S / N$ per pixel in the continuum at $6600 \AA$ is $\sim 20,15$, and 10 for the nucleus and objects $\mathrm{B}$ and $\mathrm{C}$, respectively. To obtain pure emission-line spectra, we performed starlight subtraction using observations of template stars observed with the same ESI configuration. (See Barth et al. 2008 for a detailed description of the reduction, calibration, and starlight-subtraction procedures.) The starlight-subtracted spectra of the three nuclei are shown in Figure 2] For object $\mathrm{C}$, the only detected emission lines are $\mathrm{H} \alpha$ and [N II] $\lambda 6583$.

\section{RESULTS AND DISCUSSION}

The starlight subtraction procedure yields radial velocities of $c z=8325 \pm 9,8131 \pm 6$, and $8145 \pm 6 \mathrm{~km} \mathrm{~s}^{-1}$ for the primary nucleus and objects $\mathrm{B}$ and $\mathrm{C}$, respectively. This confirms the physical association between the three objects and

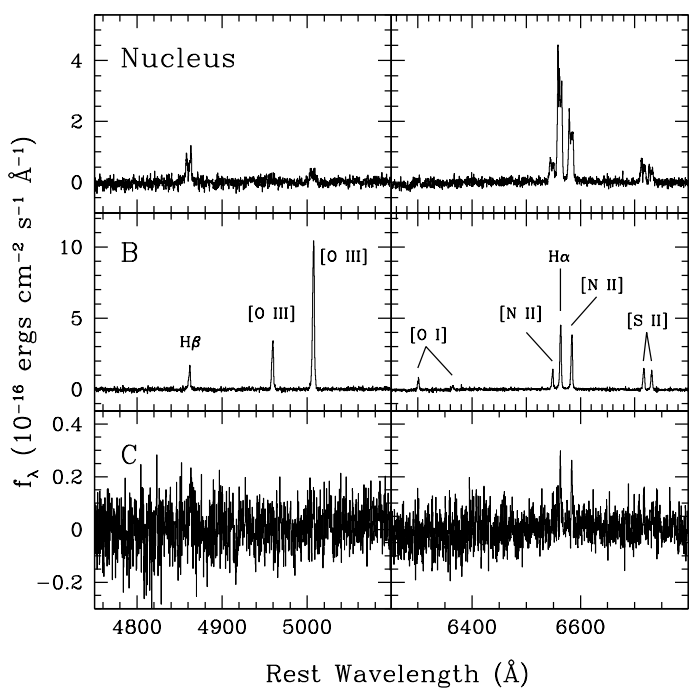

FIG. 2.- Portions of the starlight-subtracted Keck spectra of the three nuclei.

rules out a chance projection of unrelated sources.

Emission-line fluxes were measured by direct integration of the starlight-subtracted spectra; the key diagnostic flux ratios are listed in Table 1. Figure 3 displays the emissionline flux ratios of $[\mathrm{O}$ III] $] / \mathrm{H} \beta$ and $[\mathrm{N} \mathrm{II]} / \mathrm{H} \alpha$ in a Baldwin et al. (1981) diagnostic diagram, in comparison with galaxies from the Kauffmann et al. (2003) sample. The Keck spectrum of object B confirms its classification as a Seyfert 2, as it falls within the Seyfert branch of the diagnostic diagram. Its [O III] $\lambda 5007$ emission line has FWHM $=116 \mathrm{~km} \mathrm{~s}^{-1}$, similar to the low [O III] linewidths seen in other Seyfert 2 nuclei having low-mass host galaxies (Barth et al. 2008).

In the $[\mathrm{O}$ III $] / \mathrm{H} \beta$ vs. [N II] $/ \mathrm{H} \alpha$ diagram, the primary nucleus falls in the region of LINER/H II "composite" or "transition" objects, close to the boundary with $\mathrm{H}$ II nuclei, following the Kewley et al. (2006) classification scheme. However, we note that the $[\mathrm{O} \mathrm{I}] / \mathrm{H} \alpha$ and $[\mathrm{S} \mathrm{II}] / \mathrm{H} \alpha$ ratios at the nucleus are consistent with classification as an H II nucleus (Ho et al. 1997; Kewley et al. 2006). Thus, the classification of the nucleus is somewhat ambiguous. The narrow-line profiles at the nucleus are double-peaked, with a peak-to-peak velocity separation of $310 \mathrm{~km} \mathrm{~s}^{-1}$. This velocity splitting could arise from either radial motion or rotation; similar double-peaked profiles due to the rotation of a circumnuclear disk are seen, for example, in the transition-type nucleus of NGC 3245 (Ho et al. 1995).

For the weak emission lines in object $\mathrm{C}$, we find $[\mathrm{N} \mathrm{II]} / \mathrm{H} \alpha$ $=0.93 \pm 0.27$, indicated as a shaded band in Figure 3 . The nearly-equal strengths of [N II] and $\mathrm{H} \alpha$ are inconsistent with an $\mathrm{H}$ II region origin for the emission lines. Given the nondetection of [O III] $\lambda 5007$ emission, the most likely classification for this object is a LINER or LINER/H II composite. The $\mathrm{H} \alpha$ equivalent width is only $\sim 1 \AA$.

While the optical data identify object B as an AGN with a high degree of confidence, the source of ionizing photons in the nucleus and object $\mathrm{C}$ is less certain. In the $\mathrm{ab}-$ sence of definitive AGN signatures such as broad-line emission, very weak LINER-type emission lines such as those seen in object $\mathrm{C}$ could in principle result from photoionization of diffuse gas by sources unrelated to an AGN, such as post-AGB stars (Binette et al. 1994) or X-ray binaries. Indeed, Chandra observations have revealed that some nearby "Type 2" LINERs do not have a central AGN-like point source 
TABLE 1

EMISSION-LINE RATIOS

\begin{tabular}{lcccc}
\hline \hline Object & {$[\mathrm{O}$ III $] / \mathrm{H} \beta$} & {$[\mathrm{O} \mathrm{I}] / \mathrm{H} \alpha$} & {$[\mathrm{N} \mathrm{II}] / \mathrm{H} \alpha$} & {$[\mathrm{S} \mathrm{II}] / \mathrm{H} \alpha$} \\
\hline Nucleus & $0.38 \pm 0.04$ & $0.05 \pm 0.01$ & $0.54 \pm 0.02$ & $0.31 \pm 0.01$ \\
B & $6.6 \pm 0.2$ & $0.20 \pm 0.01$ & $0.85 \pm 0.02$ & $0.66 \pm 0.02$ \\
$\mathrm{C}$ & $\cdots$ & $<0.25$ & $0.93 \pm 0.27$ & $<0.6$ \\
\hline
\end{tabular}

Note. - Forbidden-line measurements refer to [O III] $\lambda 5007$, [O I] $\lambda 6300$, [N II] $\lambda 6583$, and [S II] $\lambda \lambda 6716,6731$.

at all, but do have X-ray emission dominated by a circumnuclear population of X-ray binaries (Eracleous et al. 2002; Flohic et al. 2006). As for the primary nucleus, the source of ionization in LINER/H II transition nuclei has been the subject of much debate (see $\mathrm{Ho}$ 2008). A likely interpretation is that they are composite systems containing both a weak AGN and H II regions ionized by hot stars (e.g., Ho et al. 1993; Véron et al. 1997; Kewley et al. 2006). Shock heating could play some role in the narrow-line region excitation as well (e.g., Dopita \& Sutherland 1995; Contini \& Viegas 2001). Summarizing results from X-ray surveys of nearby galaxies, $\mathrm{Ho}(2008)$ concludes that the majority of transition nuclei (perhaps $\sim 75 \%$ ) do contain AGNs. Thus, the optical spectra of the nucleus and object $\mathrm{C}$ can be interpreted as possibly containing low-luminosity accretion-powered AGNs, but the optical data do not provide conclusive evidence for the presence of AGNs in these objects. A search for X-ray sources in NGC 3341 would potentially provide the best constraints on the AGN content in this galaxy.

NGC 3341 is not detected in the ROSAT All-Sky Survey, and no source corresponding to its position is listed in the ROSAT Faint Source Catalog (Voges et al. 2000). We estimate an upper limit of 0.02 counts s$^{-1}$ in the ROSAT image, corresponding to an upper limit of $L(0.1-2 \mathrm{keV})<5 \times 10^{41}$ ergs $\mathrm{s}^{-1}$ for an unabsorbed source with a photon index of $\Gamma=1.8$. Using typical ratios of X-ray to optical emission-line flux for nearby low-luminosity AGNs (Ho 2008), the expected luminosity of each of the three nuclei would be at least an order of magnitude below the ROSAT upper limit. Thus, much deeper observations would be needed in order to search for $\mathrm{X}$-ray sources in this system. The primary nucleus is a radio source, detected in the FIRST survey (Becker et al. 1995) with a flux density of $4.8 \mathrm{mJy}$ and deconvolved major and minor axis sizes of $5 . " 5 \times 5$." 0 .

We measured the stellar velocity dispersions of the three nuclei following the direct-fitting methods described by Barth et al. (2002), by fitting the spectra over the restricted wavelength range 5020-5500 $\AA$ containing the strong $\mathrm{Mg} b$ and Fe5270 features. We find $\sigma_{\star}=182 \pm 14,70 \pm 7$, and $44 \pm 6$ $\mathrm{km} \mathrm{s}^{-1}$ for the nucleus and objects B and C, respectively. If these objects follow the $M_{\mathrm{BH}}-\sigma$ correlation for quiescent galaxies of Tremaine et al. (2002), the expected black hole masses would be $9 \times 10^{7}, 2 \times 10^{6}$, and $3 \times 10^{5} M_{\odot}$ in the three nuclei. Heckman et al. (2004) show that a typical [O III] bolometric correction for Seyfert galaxies is $L_{\mathrm{bol}} / L([\mathrm{O} \mathrm{III}]) \approx 3500$ (with a scatter of approximately $0.38 \mathrm{dex}$ ). With this bolometric correction, object B would have $L_{\mathrm{bol}} / L_{\mathrm{Edd}} \approx 0.1$.

From the 2MASS (Skrutskie et al. 2006) point source and extended source catalogs, the $K_{s}$-band magnitudes of the primary galaxy and objects B and C are 10.78, 14.26, and 15.07 mag, respectively. To estimate the stellar mass ratio of the merger, we use $K$-band mass-to-light ratios from Bell et al. (2003), computed using reddening-corrected optical colors from SDSS. Following the Bell et al. (2003) prescriptions, the

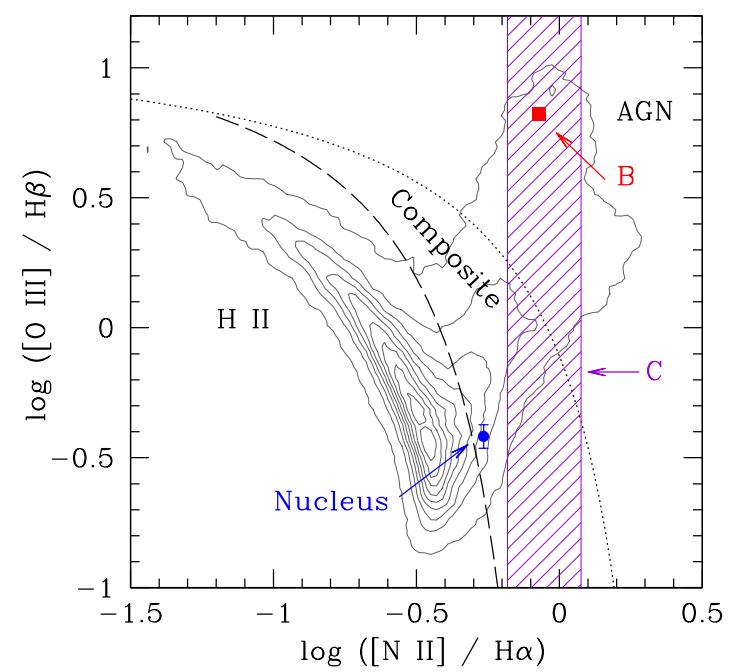

FIG. 3.- Diagnostic diagram showing the emission-line ratios of the three nuclei. Black contours denote emission-line galaxies from the Kauffmann et al. (2003) sample. The dashed and dotted curves represent the classification criteria of Kewley et al. (2006), in which H II nuclei fall below and to the left of the dashed curve, AGNs lie above and to the right of the dotted curve, and composite objects lie in the intermediate region. The primary nucleus of NGC 3341 is shown as a blue circle, and object B as a red square. For object $\mathrm{B}$ and for the $[\mathrm{N} \mathrm{II}] / \mathrm{H} \alpha$ ratio at the primary nucleus, the error bars are smaller than the plot symbols. The violet shaded band gives the $1 \sigma$ uncertainty range for the $[\mathrm{N} \mathrm{II}] / \mathrm{H} \alpha$ ratio of object $\mathrm{C}$.

primary galaxy and objects $\mathrm{B}$ and $\mathrm{C}$ each have $M / L_{K} \approx 0.85$. This yields a stellar mass of $\sim 1 \times 10^{11} M_{\odot}$ for the primary galaxy and a stellar mass ratio of 50:2:1 for the merging system (primary:B:C). We note, however, that this mass ratio does not account for any stellar mass already stripped off of objects $\mathrm{B}$ and $\mathrm{C}$ during the merger. For a more detailed examination of the structure of the merging system, higherresolution imaging would be needed. Hubble Space Telescope data could clarify whether the offset nuclei were originally spheroidal or elliptical galaxies, or the bulges or pseudobulges of low-mass disk galaxies.

It is particularly interesting to find nuclear activity in at least one of the secondary nuclei in this system, since the AGN fraction in low-mass galaxies is known to be very small overall (Kauffmann et al. 2003; Greene \& Ho 2007). Furthermore, the black hole occupation fraction in dwarf galaxies is expected to be well below unity if the efficiency for black hole seed formation is small in low-mass halos at high redshift (Volonteri et al. 2008), or if gravity-wave kicks during binary black hole coalescence can efficiently expel black holes from dwarf galaxies (Merritt et al. 2004). There are examples of AGNs with host galaxy luminosities similar to objects B and C, such as POX 52 (Barth et al. 2004), but they are predominantly isolated systems not involved in strong interactions. Simulations have shown that minor mergers can drive radial inflows of gas to the center of the primary galaxy, potentially triggering AGN accretion episodes (Hernquist \& Mihos 1995), but there is little if any statistical evidence for enhanced AGN activity in the secondary galaxies of minor interaction systems. In a study of close (separation $<50 h^{-1} \mathrm{kpc}$ ) galaxy pairs in SDSS, Woods \& Geller (2007) found that in minor interactions with $\Delta m>2 \mathrm{mag}$, there was a strong increase in the AGN fraction for the primary galaxies (relative to a matched field galaxy control sample) but there was no detectable enhancement in the AGN fraction of the secondary 
galaxies in the pairs relative to a matched control sample. In view of the properties of NGC 3341, we speculate that any enhancement in the AGN fueling rate in the secondary might occur at very small separations $(\lesssim 10 \mathrm{kpc})$ that are difficult to probe in statistical surveys.

This unusual AGN host galaxy was discovered because the SDSS fortuitously selected object B as the one spectroscopic target in this field. If the primary nucleus had been targeted instead, the galaxy would have appeared unremarkable, and follow-up observations of objects $\mathrm{B}$ and $\mathrm{C}$ would have been unlikely. What are the prospects for detection of offset AGNs in other nearby galaxies? King \& Dehnen (2005) argued that some of the most extreme ultraluminous X-ray (ULX) sources are likely to be the nuclei of captured satellite galaxies undergoing AGN accretion episodes, rather than being X-ray binaries with intermediate-mass black holes. While no ULX sources are currently known to have an origin in captured satellites, the discovery of NGC 3341B in the SDSS suggests that optical selection can be at least as efficient as X-ray selection for discovery of off-nuclear, accreting black holes from satellite galaxies in minor mergers. However, optical selection depends crucially on the ability to identify the nucleus of the secondary galaxy in imaging data. As discussed by Woods et al. (2006), there are severe observational biases that make it very difficult to identify satellite galaxies involved in minor interactions $(\Delta m>2 \mathrm{mag})$ at very close separations of $\lesssim 10 \mathrm{kpc}$ from the primary galaxy. Furthermore, at separations of a few kpc, satellite galaxies may be so severely disrupted that they no longer have identifiable nuclei, and offset, low-luminosity AGNs not embedded in stellar nuclei would likely be inconspicuous in optical surveys. Nevertheless, further searches for systems like NGC 3341 containing low-mass companions at small separations could provide new constraints on the demographics of dual AGNs and the efficiency of AGN fueling in minor mergers.

Research by A.J.B. and M.C.B. is supported by NSF grant AST-0548198. Data presented herein were obtained at the W.M. Keck Observatory, which is operated as a scientific partnership among Caltech, the University of California, and NASA. The Observatory was made possible by the generous financial support of the W.M. Keck Foundation. The authors wish to recognize and acknowledge the very significant cultural role and reverence that the summit of Mauna Kea has always had within the indigenous Hawaiian community. Funding for the SDSS and SDSS-II has been provided by the Alfred P. Sloan Foundation, the Participating Institutions, the National Science Foundation, the U.S. Department of Energy, the National Aeronautics and Space Administration, the Japanese Monbukagakusho, the Max Planck Society, and the Higher Education Funding Council for England. This publication makes use of data products from the Two Micron All Sky Survey, which is a joint project of the University of Massachusetts and the Infrared Processing and Analysis Center/California Institute of Technology, funded by NASA and the NSF.

\section{REFERENCES}

Baldwin, J. A., Phillips, M. M., \& Terlevich, R. 1981, PASP, 93, 5

Ballo, L., Braito, V., Della Ceca, R., Maraschi, L., Tavecchio, F., \& Dadina, M. 2004, ApJ, 600, 634

Barth, A. J., Greene, J. E., \& Ho, L. C. 2008, AJ, in press

Barth, A. J., Ho, L. C., Rutledge, R. E., \& Sargent, W. L. W. 2004, ApJ, 607, 90

Barth, A. J., Ho, L. C., \& Sargent, W. L. W. 2002, AJ, 124, 2607

Becker, R. H., White, R. L., \& Helfand, D. J. 1995, ApJ, 450, 559

Bell, E. F., McIntosh, D. H., Katz, N., \& Weinberg, M. D. 2003, ApJS, 149, 289

Bianchi, S., Chiaberge, M., Piconcelli, E., Guainazzi, M., \& Matt, G. 2008, MNRAS, 369

Binette, L., Magris, C. G., Stasińska, G., \& Bruzual, A. G. 1994, A\&A, 292, 13

Contini, M., \& Viegas, S. M. 2001, ApJS, 132, 211

Dopita, M. A., \& Sutherland, R. S. 1995, ApJ, 455, 468

Eracleous, M., Shields, J. C., Chartas, G., \& Moran, E. C. 2002, ApJ, 565, 108

Flohic, H. M. L. G., Eracleous, M., Chartas, G., Shields, J. C., \& Moran, E. C. 2006, ApJ, 647, 140

Gerke, B. F., et al. 2007, ApJ, 660, L23

Greene, J. E., \& Ho, L. C. 2007, ApJ, 667, 131

Guainazzi, M., Piconcelli, E., Jiménez-Bailón, E., \& Matt, G. 2005, A\&A, 429, L9

Heckman, T. M., Kauffmann, G., Brinchmann, J., Charlot, S., Tremonti, C., \& White, S. D. M. 2004, ApJ, 613, 109

Hernquist, L., \& Mihos, J. C. 1995, ApJ, 448, 41

Ho, L. C. 2008, ARA\&A, in press (arXiv:0803.2268)
Ho, L. C., Filippenko, A. V., \& Sargent, W. L. W. 1993, ApJ, 417, 63 Ho, L. C., Filippenko, A. V., \& Sargent, W. L.W. 1995, ApJS, 98, 477 Ho, L. C., Filippenko, A. V., \& Sargent, W. L. W. 1997, ApJS, 112, 315 Kauffmann, G., et al. 2003, MNRAS, 346, 1055

Kewley, L. J., Groves, B., Kauffmann, G., \& Heckman, T. 2006, MNRAS, 372,961

King, A. R., \& Dehnen, W. 2005, MNRAS, 357, 275

Komossa, S. 2003a, in The Astrophysics of Gravitational Wave Sources (AIP Conference Proceeding), 686, 161

Komossa, S., Burwitz, V., Hasinger, G., Predehl, P., Kaastra, J. S., \& Ikebe, Y. 2003b, ApJ, 582, L15

Merritt, D., \& Milosavljević, M. 2005, Living Reviews in Relativity, 8, 8

Merritt, D., Milosavljević, M., Favata, M., Hughes, S. A., \& Holz, D. E. 2004, ApJ, 607, L9

Owen, F. N., O’Dea, C. P., Inoue, M., \& Eilek, J. A. 1985, ApJ, 294, L85

Sheinis, A. I., Bolte, M., Epps, H. W., Kibrick, R. I., Miller, J. S., Radovan,

M. V., Bigelow, B. C., \& Sutin, B. M. 2002, PASP, 114, 851

Skrutskie, M. F., et al. 2006, AJ, 131, 1163

Tremaine, S., et al. 2002, ApJ, 574, 740

Valluri, M., Ferrarese, L., Merritt, D., \& Joseph, C. L. 2005, ApJ, 628, 137

Véron, P., Gonçalves, A. C., \& Véron-Cetty, M.-P. 1997, A\&A, 319, 52

Voges, W., et al. 2000, IAU Circ., 7432, 3

Volonteri, M., Lodato, G., \& Natarajan, P. 2008, MNRAS, 383, 1079

Woods, D. F., \& Geller, M. J. 2007, AJ, 134, 527

Woods, D. F., Geller, M. J., \& Barton, E. J. 2006, AJ, 132, 197 